\title{
Low cost, Autonomous and Wireless Enabled Liquid Level Sensor Based on a Multi-Segmented Polymer Optical Fiber
}

\author{
Dimitrios DIMAS ${ }^{1.2 .3}$, Serafeim KATSIKAS ${ }^{3}$, Anthony BOUCOUVALAS ${ }^{2}$, Christos RIZIOTIS ${ }^{1}$ \\ ${ }^{1}$ The National Hellenic Research Foundation, Theoretical and Physical Chemistry Institute, 48 Vassileos \\ Constantinou Avenue, Athens 11635, Greece \\ ${ }^{2}$ University of Peloponnese, Dpt of Telecommunications Science and Technology, Tripoli 22 100, Greece \\ ${ }^{3}$ Prisma Electronics SA, El. Venizelou 126, Nea Smyrni, 17123 Athens, Greece
}

\begin{abstract}
The optical sensors' technology has reached a quite mature level that could enable its successful integration in real demanding applications further to the lab environment. However the lack of effective standardization together with the quite broad range of photonics technology has delayed considerably their real employment. From the applications side the need of effective autonomous and wireless sensing networks for industrial applications sets very strict requirements in the complexity and cost of optical sensors in order to make their integration in working environments viable.

Having identified in this context suitable photonic architectures for effective employment we propose here a new simple design, based on Polymer Optical Fiber, of very low implementation cost, as an effective liquid level sensor. POF based sensors lately are placed amongst the favored solutions offering significant advantages over silica fibers and at much reduced cost and complexity. Liquid level sensing is of continuous demand in a range of industrial applications from chemical to mechanical areas. Level sensors based on photonic operation offers a new solution of added value for measuring in industrial environments with excessive electromagnetic noise, interference and suitable also for measuring flammable or explosive liquids (alcohols, gasoline, diesel, etc) as no electric currents are present in the detection. The design is based on a multi-segmented polymer optical fiber (POF) which forms a relatively low loss discontinuous channel able to effectively sense presence of a large variety of liquids and at a long leveling length range. The low cost and low power consumption suggest this solution as an autonomous sensor and also wireless enabled.
\end{abstract}

\section{Introduction}

Wireless Sensor Networks (WSNs) consist of various nodes called sensor nodes. Due to their basic characteristics of autonomy, low power consumption, data processing and storing capability and wireless communication they are also called smart sensors. A sensor node includes the sensing units, an embedded microprocessor that processes the data retrieved from the sensing units, a memory for local storing when needed, an RF communication module, the required power supply unit, usually a set of $1.5 \mathrm{~V}$ batteries and a dedicated operating system and the appropriate middleware for flexible application development [2]. The easy deployment and recollection, the absence of wires for communication needs, the data processing and storing capabilities, the ability for distributed sensing and the fact that smart sensors are autonomous and rather cheap are among the main advantages when using wireless smart sensors.
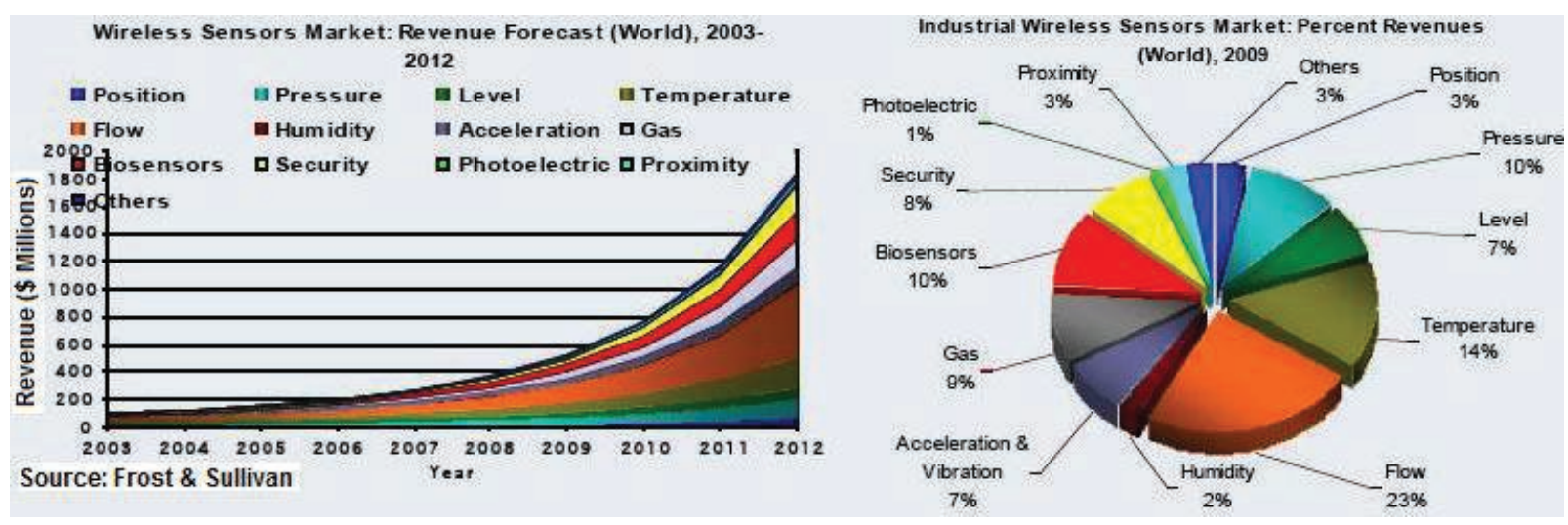

Figure 1: Wireless sensor market actual revenue since 2002 and projected forecast for 2009-2012 (Source: Frost \& Sullivan report by Thusu, 2010)

Temperature, pressure, flow and level are the most measured parameters with wireless sensors with revenue shares of $14.3 \%, 9.9 \%, 23.6 \%$ and $6.6 \%$ respectively, see figure 1 . Level sensors are used to 
monitor material levels and to track the rate of material usage in remote locations. Wireless level sensing is used mostly in industrial applications such as tank inventory monitoring and logistics, equipment preventive maintenance, real-time truck scheduling and routing. The cost of remote tank inventory monitoring can be lowered significantly by using a wireless level monitoring system [3]. They can also be used for liquid level or flood sensing for environmental purposes (rivers, canals), for industrial applications like monitoring flammable or explosive liquids in chemical factories, refineries, chemical stores and warehouses, fuel tanks and in pipelines for leakage detection.

\section{Plastic Optical Fiber Based Sensors}

Photonic-based sensors show rather attractive characteristics like small size, light weight, immunity to electromagnetic interference (EMI), passive (all dielectric) composition, high temperature performance, large bandwidth, low power, high sensitivity, environmental ruggedness, the ability of distributed sensing and the multiplexing capabilities [4]. Due to all of these characteristics, photonic sensors are great candidates for adoption in Wireless Sensor Networks, but some important requirements like low power consumption and light intensity-based interrogation system have to be fulfilled [5].

Polymer Optic Fibers (POFs) is a type of fiber that is mainly used for short-length communication applications, due to the great light attenuation that they present, although recent advances of new fabrication methods and special polymer materials have greatly reduced the fiber attenuation. On the contrary, polymer optical fibers have inherent advantages, such as the much larger diameter $(0.25-3 \mathrm{~mm})$, the reduced cost of the fiber and the lower cost of the components needed. They also exhibit excellent chemical and weather resistance and do not easily corrode when in direct contact with a liquid medium [6]. They are also much easier to handle, terminate, connect and are more flexible, characteristics that are important for sensing applications, where in many cases the fiber is side-polished or cut in order to let the core of the fiber in direct contact with the sensing region.

\section{Liquid Level Sensor Design, Configuration and Results}

POF based liquid level sensors have been recently demonstrated employing an approach of bending and careful side polishing of fiber's cladding or even complete removal of it, inducing thus differential optical transmission changes when the fiber is immersed in liquids. Two similar approaches demonstrated one of a spiral coil shaped POF [7] polished at each turn and another based on a number of bent and polished POF segments serving as measurement points [8]. Given the small thickness (20 um) of POF's cladding, special attention should be taken for the careful and reproducible treatment of the fiber implied thus a relatively costly procedure.

We propose and demonstrate here a simple design based on a multi segmented POF that exhibits better performance, at lower cost and complexity, and can be integrated in an autonomous wireless sensing node.

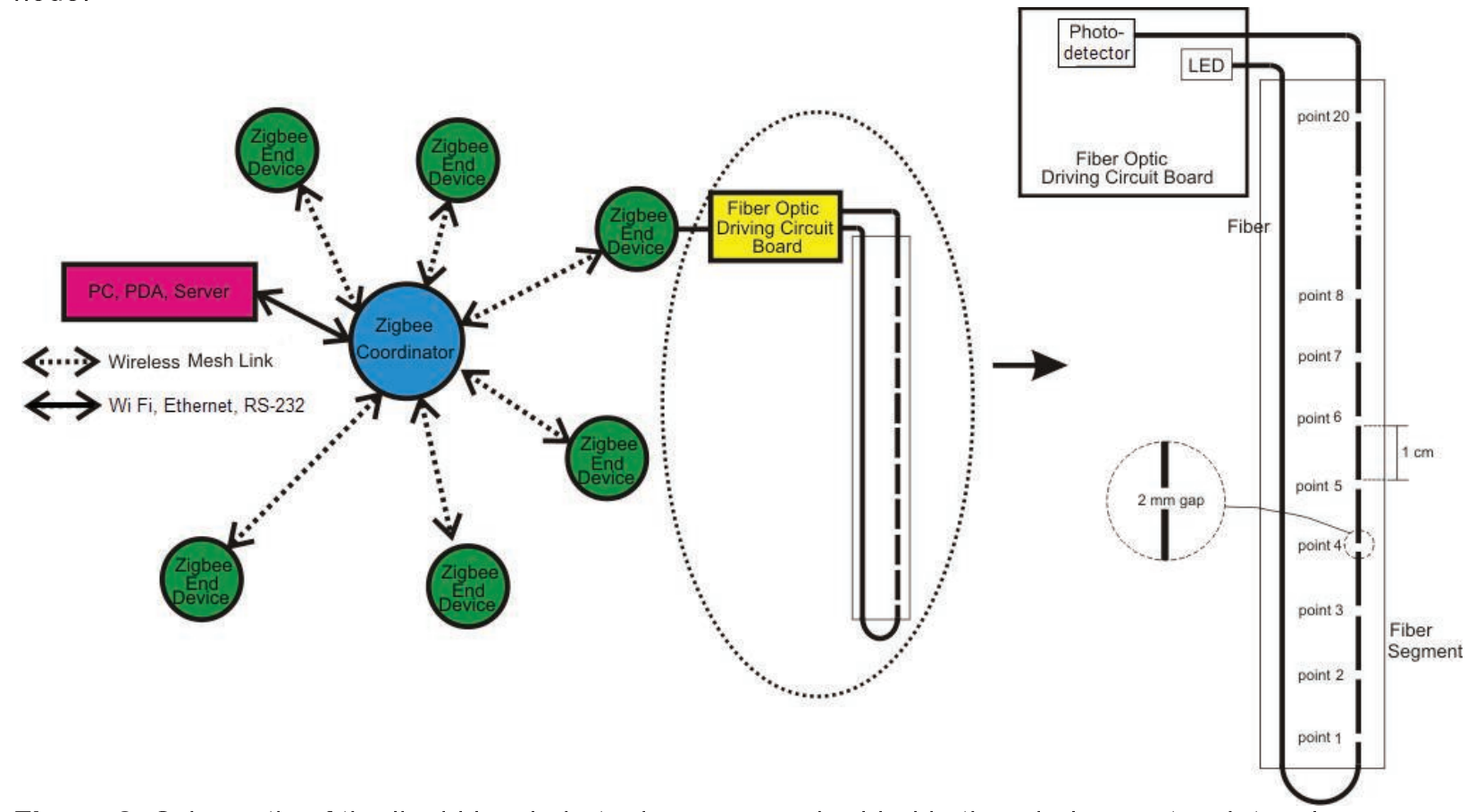

Figure 2: Schematic of the liquid level photonic sensor embedded in the wireless network topology 
The sensor that can be seen in figure 2 was implemented by creating a discontinuous POF transmission system by aligning fiber segments with gaps in between and by locating them quickly and accurately on a suitable V-groove channel. The sensor consists of the Fiber Optic Driver Circuit Board-FODCB that has a cheap LED of $200 \mu \mathrm{W}$ at $650 \mathrm{~nm}$, a photodetector, an amplifier for amplifying the measured voltage and the appropriate electronic driving circuits. The output of the amplifier is connected to the A/D converter of the wireless sensor node for further processing. The POF used was a typical PMMA POF with core and cladding diameter of $1 \mathrm{~mm}$. The FODCB is supplied by $2 \mathrm{AA}$ batteries of $3 \mathrm{~V}$ in total. The prototype of the FODCB can be seen on figure 3 .

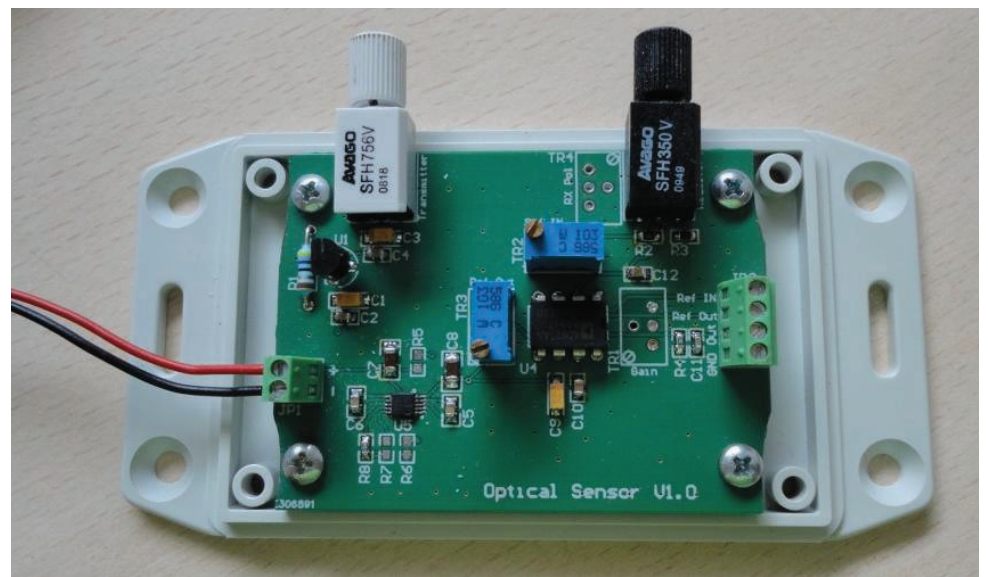

Figure 3: The prototype FODCB with low power consumption for autonomous operation

When the sensor is immersed in a liquid the light propagating path properties change, adjusting accordingly the total light delivered to the photodetector. The level measuring system has an intrinsic discrete behavior making the sensor robust to any imperfections. By adjusting the length of the segments the measured leveling/depth range can be simply expanded to any required length. Despite the noncontinuous optical channel design -and the anticipated high transmission loss- the sensor exhibits rather low optical loss reaching thus easily the maximum number (\#18 points) of measuring points demonstrated in previous works [7], showing also an adequate discrimination signal strength between different liquid levels. Due to the fiber gaps also, the discrimination between "Full' and "Empty" condition at each point was very fast $(<2 \mathrm{sec})$ in contrast to the polished fiber sensor where any residue wetting of the polished fiber was inducing wrong interpretation.

The sensor characterized in a variety of liquids (namely water, automobile gasoline and diesel) at room temperature showing stable performance. The results of the measured liquids can be seen in figure 4 .

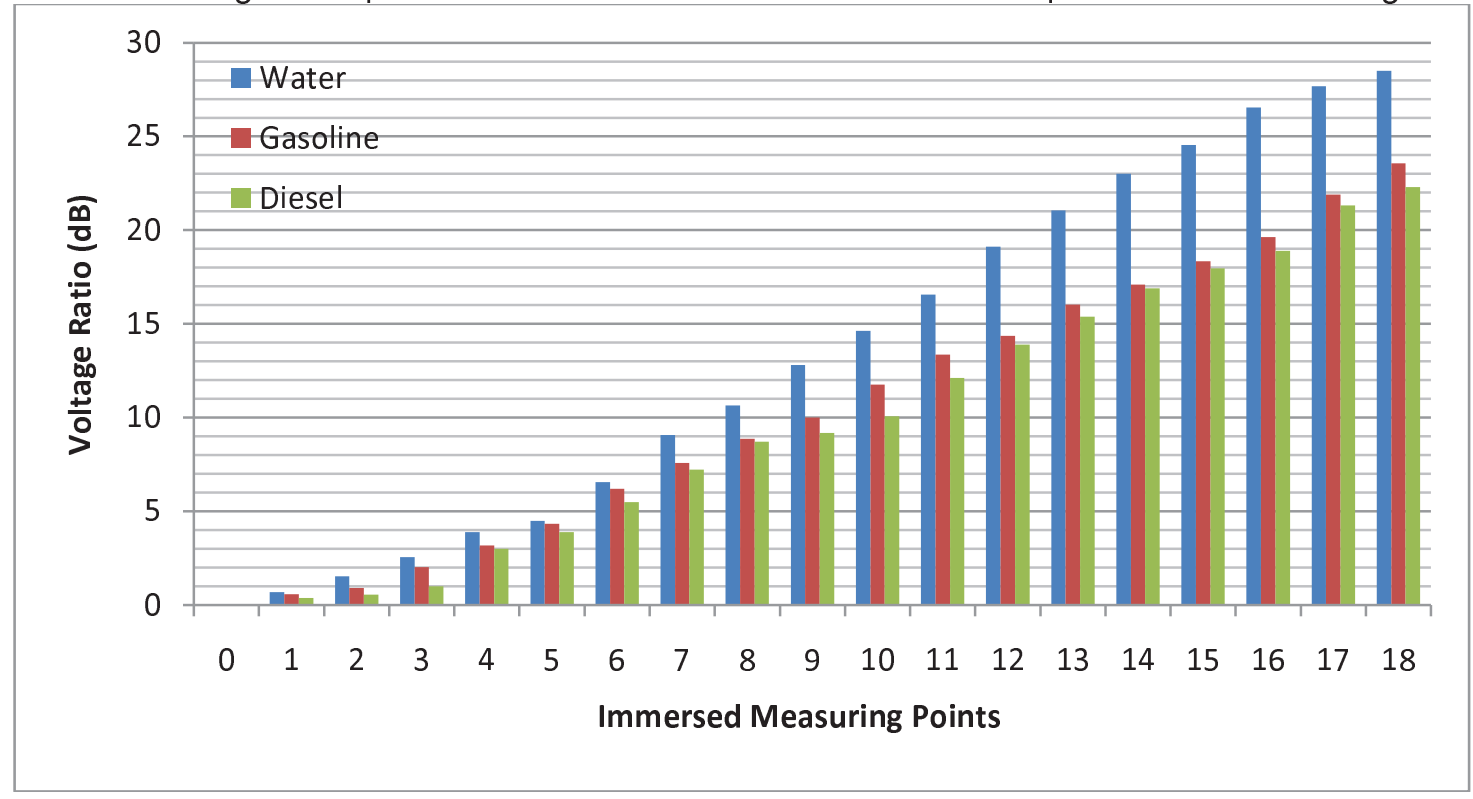

Figure 4: Graph showing the liquid level sensor response to three different liquids (water, automobile gasoline and diesel) 
The $y$-axis shows the ratio in $\mathrm{dB}$ of the measured voltage at the ends of the photodetector when various measuring points have been immersed in a liquid compared to the voltage when no points are in the liquid. As can be seen the sensor shows an almost linear behavior in logarithmic scale of voltage ratio to the count of measured points.

\section{Long Range Liquid Level Sensor Design, Configuration and Results}

As abovementioned by adjusting the length of the segments the measured leveling range could be simply expanded to any required length, as long as enough light is propagated along the fiber. By using appropriate POF segment length this single POF channel could serve as a long range level sensor for leveling lengths in the order of $100 \mathrm{~m}$ given the suitable instrumentation and amplification of the signal. Equivalently this long range operation could be seen also as a distributed liquid sensing system forming a large area flood monitoring / warning system. Alternative approaches use a number of interconnected wireless sensor nodes, but with additional cost compared to this distributed sensor system [9], since each measuring point requires a single dedicated wireless sensor node. The presented below flood monitoring results are based on a different than the previously presented segmented POF approach, in order to simplify, speed up and facilitate the experimental demonstration as the validation of long length range level measurements. We have experimentally confirmed that the losses of segmented section of the fiber are of the same order to the losses induced by a vertical to the fiber axis cut of 200 micrometers . So, each measuring point of the POF was modified by inducing an accurate thin cut (with a hot knife) of 200 micrometers depth vertical to the axis of the fiber, see figure 5.

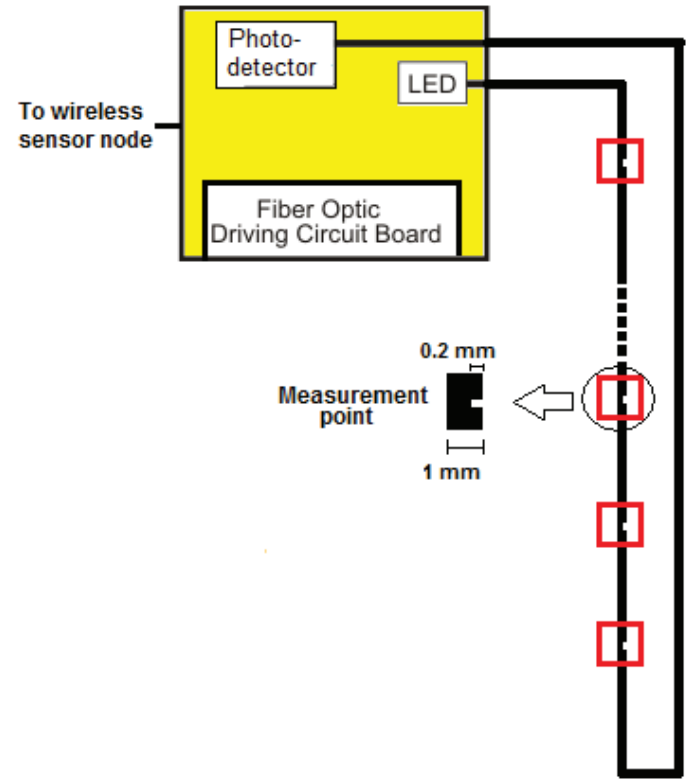

Figure 5: Schematic of the distributed flood monitoring sensor

For implementing the long range level sensor 50 meter of typical PMMA fiber and a still low cost / low power $1 \mathrm{~mW}$ LED source were used. Experiments showed that is completely feasible to monitor changes of liquid leakage for more that 30 measuring points distributed along the fiber. The limitation of the length presented here is due to the limited available length of the POF. However special and still low cost (as perfluorinated fiber -CYTOP) could be used for extending further the length range. This induced 'mark' in the fiber allowed the exposure of the core and the associated optical field to the liquid measurand inducing thus changes in the propagation loss and so at the measured signal of the photodetector, as shown in figure 6. 


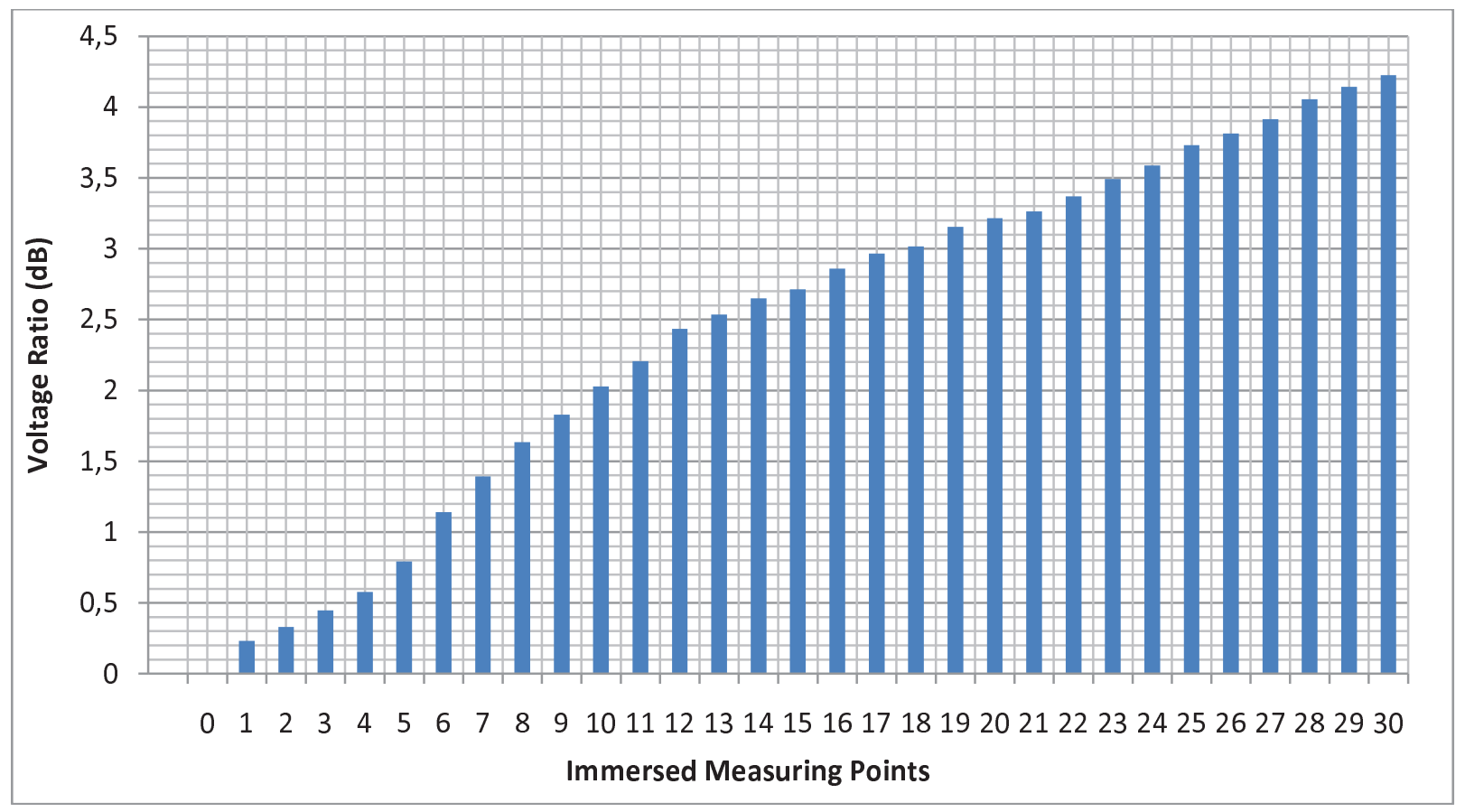

Figure 6: Graph showing the distributed flood monitoring sensor response to water

The $y$-axis shows, as in the graph of the liquid level sensor, the ratio in $\mathrm{dB}$ of the measured voltage at the ends of the photodetector when various measuring points have been immersed in water compared to the voltage when no points are in the liquid. The sensor shows an almost linear behavior in logarithmic scale of voltage ratio to the count of immersed points. Some small discrepancies in the measured values could be associated to the non-optimized setup for marking the fiber accurately at constant depth $0.2 \mathrm{~mm}$.

As can be understood from figures 4 and 6 when the first measurement points are immersed into a liquid, the difference of the photodetector's voltage is rather small but as more points are immersed the differences are getting higher, which is rather a logarithmic behavior. This is not a problem, because by choosing an appropriate amplifier for the FODCB, for example a logarithmic amplifier this logarithmic behavior can be compensated, so the output voltage of the FODCB will show a linear response to the number of the immersed measurement points. The changes were reversible after the drying of the liquid at those points.

While the "marking" of the fiber for inscripting the liquid sensing points by using the hot knife technique could be a quite accurate and simple technique we have identified an alternative method that could possibly allow more parameterization with more enhanced functionality. We are proposing the use of an excimer pulsed laser for accurately etching/structuring the fiber and provide here preliminary results on the technique. We have adopted the use of an ArF $193 \mathrm{~nm}$ pulsed excimer laser which allows the accurate marking by adjusting a number of parameters such as pulse count, focussing, writing speed etc . The system is essentially a Laser micromachining station with nanopositioning translation stages that allows the direct write/definition of structures.

An indicative groove defined on a POF fiber by this technique is demonstrated in figure 7. By adjusting the writing conditions the depth of the structure and the length could be accordingly adjusted achieving thus the required sensitivity to liquid detection and also managing the associated trade off of the induced optical loss due to the perturbation. Characterization of such a laser "marked" POF based level sensor is currently under way. 


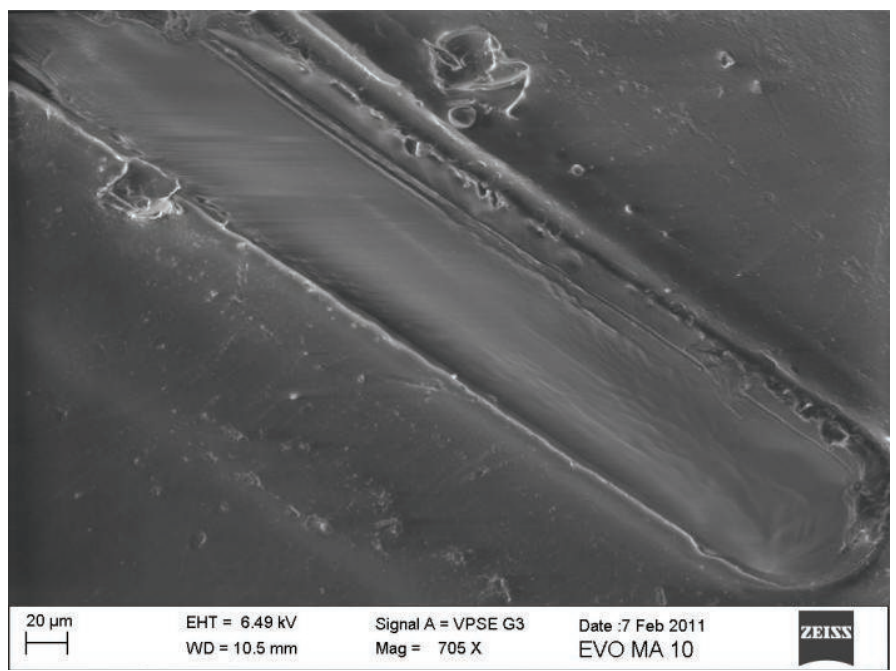

Figure 7: SEM image of the groove induced by a 193nm Laser radiation on a PMMA fiber.

\section{Conclusions}

We have demonstrated a novel low cost approach towards the effective incorporation of polymer optical fibers sensors technology in autonomous wireless sensing nodes and networks. The employments of photonics in the challenging area of liquid level sensing enhances the applications' functionality by providing a platform of minimal electromagnetic interferences and EM noise, securing in general electrical safety characteristics and allowing also the safe operation and level monitoring in flammable environments. The multisegmented or the partially perturbed POF, forms a discontinuous monitoring channel able to monitor liquid leveling in a range of liquids and in a quite long range of distance, by deploying low cost and low power optical sources and receivers. These low cost and low consumption characteristics make the proposed sensing system autonomous and wireless enabled, providing thus the extra networking flexibility and functionality.

\section{Acknowledgements}

This work is partially funded by the R\&D project entitled: "Development of Innovative sensor systems offering distributed intelligence - MEMSENSE", which is funded by "ESPA-ETAK 2007-2013", and also by the project SESAMO (SEnsors for StructurAl Monitoring) of the European Defense Agency (EDA) with Grant Number A-0931-RT-GC.

\section{References}

[1] C. Riziotis, V. Pruneri, P.G.R. Smith, A.V. Vasilakos, "Fiber and Integrated Waveguide-Based Optical Sensors", Journal of Sensors, vol. 2009, Article ID 171748.

[2] C. Emmanouilidis, S. Katsikas, P. Pistofidis, C. Giordamlis, "A Wireless Sensing Development Platform for Ubiquitous Condition Monitoring". COMADEM 2009, $22^{\text {nd }}$ International Congress, San Sebastian, Spain.

[3] R. Thusu, 2010. "Wireless Sensor Use Is Expanding in Industrial Applications. Sensors Magazine". Available from http://www.sensorsmag.com/networking-communications/wireless-sensor/wireless-sensor-use-isexpanding-industrial-applications-7212 [Accessed 2011/02/14]]

[4] B. Gholamzadeh, H. Nabovati. 2008. Fiber Optic Sensors. World Academy of Science, Engineering and Technology, Issue 42, pp. 297-307.

[5] C. Riziotis, D. Dimas, S. Katsikas, A.C. Boucouvalas, "Photonic sensors for autonomous wireless sensing nodes", COMADEM 2010 - Proc. 23rd International Congress. Condition Monitoring and Diagnostic Engineering Management, pp. 669-676. Nara, Japan.

[6] J. Zubia, J. Arrue. 2001. Plastic Optical Fibers: An Introduction to Their Technological Processes and Applications. Optical Fiber Technology, Volume 7, Issue 2, pp. 101-140.

[7] M. Lomer, A. Quintela, M. Lopez-Amo, J. Zubia, J. M. Lopez-Higuera. 2007. "A quasi-distributed level sensor based on a bent side-polished plastic optical fibre cable". Measurement Science and Technology 18, pp. 22612267.

[8] M. Lomer, J. Arrue, C. Jauregui, P. Aiestaran, J. Zubia, J.M. Lopez-Higuera. 2007. "Lateral polishing of bends in plastic optical fibres applied to a multipoint liquid-level measurement sensor". Sensors and Actuators A, Volume 137, Issue 1, pp. 68-73.

[9] K.S.C. Kuang, S. T. Quek, M. Maalej. 2008. "Remote flood monitoring system based on plastic optical fibres and wireless motes". Sensors and Actuators A, Volume 147, Issue 2, pp. 449-455. 